\title{
Avaliação de corpos estranhos no segmento anterior com ultrassonografia biomicroscópica
}

\author{
Evaluation of anterior segment foreign bodies with ultrasound biomicroscopy \\ Marília Freitas de Moura ${ }^{1}$, Ilka Hayashi ${ }^{1}$, Daniel Martins Rocha ${ }^{2}$, Norma Allemann ${ }^{1}$
}

\section{RESUMO}

Introdução: $O$ traumatismo ocular é a causa mais importante de perda visual no mundo, sendo um problema de saúde pública. Os corpos estranhos intraoculares são relacionados a atividades que envolvem manuseio de objetos metálicos, porém outros materiais podem estar presentes, e devem ser diagnosticados e localizados no olho. A ultrassonografia biomicroscópica é uma ferramenta complementar adequada para avaliar um corpo estranho do segmento anterior do olho.

Objetivo: Caracterizar a natureza, localização, dimensões de corpos estranhos e lesões associadas ao segmento anterior, utilizando-se ultrassonografia biomicroscópica. Método: Estudo clínico retrospectivo, por meio da análise de 7.182 prontuários dos anos de 1999 a 2008, com 59 exames de pacientes com suspeita de corpo estranho no segmento anterior ocular.

Resultados: Dentre os 59 exames, confirmou-se pela ultrassonografia biomicroscópica a presença de corpo estranho no segmento anterior em $42(71,19 \%)$ olhos de 42 pacientes. A idade dos pacientes variou entre 9 e 76 anos (média=37,4 anos). Houve predominância do sexo masculino (85,71\%). Cinco casos apresentaram dois corpos estranhos, fragmentos da mesma natureza, em diversas localizações ou não; e três casos continham incontáveis fragmentos dispostos no segmento anterior. Considerando sua localização: córnea, 11 (26\%); conjuntiva, 10 (23\%); íris, 10 (23\%); cristalino, 9 (21\%); esclera, 5 (12\%); seio camerular, 5 (12\%); corpo ciliar, 3 (7\%). Dentre as lesões associadas, identificou-se: perfuração corneana em 12 casos, laceração corneana em 2 casos, sinéquia anterior em 6 casos, aniridia traumática em 1 caso, iridotomia traumática em 3 casos, reação de câmara anterior em 10 casos e rotura de cápsula anterior do cristalino em 4 casos. Considerando a composição: metálicos, 21 (50\%); não-metálicos, 20 de vidro (48\%) e 1 de origem vegetal (2\%). O tamanho dos corpos estranhos variou de 0,09 a 2,45 mm (média: 0,84 mm).

Conclusões: A ultrassonografia biomicroscópica é útil para localizar corpos estranhos no segmento anterior. Este método de imagem pode proporcionar orientação sobre a natureza, o trajeto, a localização dos corpos estranhos e lesões associadas, facilitando o planejamento cirúrgico prévio à intervenção, evitando lesões adicionais ao olho.

Descritores: Corpos estranhos no olho/ultrassonografia; Traumatismos oculares/ ultrassonografia; Microscopia/métodos; Sensibilidade e especificidade

\section{ABSTRACT}

Introduction: Ocular trauma is considered a health care problem because is an important cause for visual impairment. Intraocular foreign bodies are related to activities involving usage of metallic objects, but other materials can be present in the eye and have to be diagnosed and localized. Ultrasound biomicroscopy is an adequate subsidiary tool to evaluate an anterior chamber intraocular foreign body.

Purpose: To characterize nature, localization, dimensions of foreign bodies and associated lesions to the anterior segment using ultrasound biomicroscopy.

Methods: Retrospective clinical study of 7,182 patient's charts submitted to ultrasound biomicroscopy examination between 1999 and 2008, totalizing 59 eyes suspected of anterior segment foreign body.

Results: Five cases presented two foreign bodies, fragments from the same nature, in different locations or not; and three cases had uncountable fragments in the anterior segment. Concerning localization: cornea, 11 (26\%); conjunctiva, 10 (23\%); iris, 10 (23\%); lens, 9 (21\%); sclera, 5 (12\%); corneal angle, 5 (12\%); ciliary body, 3 (7\%). Among the associated lesions, it was identified: corneal perforation in 12 cases, corneal laceration in 2 cases, anterior synechia in 6 cases, traumatic aniridia 1 case, traumatic iridotomy in 3 cases, anterior chamber reaction in 10 cases and rupture of the anterior lens capsule in 4 cases. Considering composition: metallic, 21 (50\%); non metallic, 20 were glass (48\%) and 1 was vegetal (2\%). The size of foreign bodies varied from 0.09 to $2.45 \mathrm{~mm}$ (average: $0.84 \mathrm{~mm}$ ).

Conclusions: Ultrasound biomicroscopy is useful to localize foreign bodies in the anterior segment. This imaging method can give orientation about composition, path, and localization of foreign bodies and associated lesions, thus facilitating therapeutic planning prior to intervention, avoiding additional lesions to the eye.

Keywords: Eyeforeign bodies/ultrasonography; Eyeinjuries/ultrasonography; Microscopy/ methods; Sensibility and specificity

\section{INTRODUÇÃO}

O traumatismo ocular é a causa mais importante de perda visual no mundo, sendo um problema de saúde pública que se deve prevenir ${ }^{(1)}$. Os acidentes com corpos estranhos são mais comuns em atividades que envolvem o manuseio de metais. Contudo, vegetais, vidro e outros materiais também podem estar presentes ${ }^{(2)}$. A correta localização de corpos estranhos intraoculares é essencial para o planejamento terapêutico ${ }^{(3,4)}$.
Dependendo da composição dos corpos estranhos, pode haver urgência de sua remoção. Materiais como ferro e cobre são tóxicos e estimulam o processo inflamatório. Vegetais predispõem ao risco de infecção, portanto, devem ser removidos com presteza. Substâncias inertes, como vidro e plástico, podem ser bem toleradas pelo organismo ${ }^{(5)}$.

A presença de corpos estranhos pode limitar ou impedir a visibilidade das estruturas oculares ${ }^{(5)}$. A ultrassonografia biomicroscópica (UBM) possui boa resolução para avaliar o segmento anterior ${ }^{(6-8)}$
Financiamento: Não houve financiamento para este estudo.

Divulgação de potenciais conflitos de interesse: M.F.Moura, Não; I.Hayashi, Não; D.M.Rocha, Não; N.Allemann, Não.

Endereço para correspondência: Norma Allemann. Av. Indianópolis, 1797 - São Paulo (SP) 04063-003 - Brazil - E-mail: norma.allemann@pobox.com 
e a superfície de um corpo estranho. A avaliação clínica permitirá decidir o momento mais seguro para realização do exame complementar, que pode ser realizado em uso de lentes de contato terapêuticas para evitar excessiva manipulação e com utilização de meios condutores líquidos estéreis para evitar adicional risco de infecção pela porta de entrada ${ }^{(7,8)}$.

À UBM, os corpos estranhos geram vários artefatos que são devidos a suas características acústicas. Uma bolha de ar absorve o som criando uma sombra, enquanto objetos densos, como metal e vidro, geram artefatos tipo "cauda de cometa" pela reflexão do som² ${ }^{(2)}$.

O objetivo deste trabalho é caracterizar, por meio da ultrassonografia biomicroscópica, os corpos estranhos do segmento anterior quanto a natureza, localização, tamanho e lesões associadas.

\section{MÉTODOS}

Estudo clínico retrospectivo, conduzido no Setor de Ultrassonografia do Departamento de Oftalmologia da Universidade Federal de São Paulo, por meio da análise de 7.182 prontuários dos anos de 1999 a 2008, dos quais foram extraídos 59 exames de pacientes com suspeita de corpo estranho no segmento anterior ocular pela UBM.

A UBM não foi realizada nos casos que apresentaram ferida aberta, devido ao risco de contaminação e de extrusão de conteúdo intraocular.

Os exames de UBM foram executados após instilação de 4 gotas de colírio de cloridrato de proximetacaína a 0,5\% e colocação de cuba de imersão entre as pálpebras do olho a ser examinado, com o paciente em decúbito dorsal. Utilizaram-se dois tipos de aparelho com transdutores de $50 \mathrm{MHz}$, dependendo do período em que os exames foram feitos. No período de 1999 a 2007, foram examinados 52 pacientes (88,13\%) com o UBM 840 (Humphrey), com imersão com solução de metilcelulose a 2\%. De 2006 a 2008, 10 exames $(16,94 \%)$ foram feitos com o equipamento UBM Vumax (Sonomed), com imersão em soro fisiológico a 0,9\%. Em 3 casos (5,07\%), realizou-se o exame com os dois aparelhos.

Foram feitos cortes ultrassonográficos axiais da câmara anterior central, seguidos de cortes radiais (Iongitudinais). Quando o corpo estranho era identificado, realizava-se a exploração do local com cortes radiais e transversais. Quando possível, realizou-se a medida das suas dimensões.

\section{RESULTADOS}

Dentre os 59 exames de UBM analisados com suspeita de corpo estranho do segmento anterior, houve reconhecimento de algum tipo de corpo estranho no segmento anterior em 42 casos $(71,19 \%)$ de 42 pacientes, enquanto que em 17 olhos (28,81\%) não se detectou a presença do mesmo. A idade dos pacientes com corpo estranho variou de 9 a 76 anos (média de 37,49 anos) e a maioria era homem $(85,71 \%)$

Dos 42 casos com corpos estranhos detectados no segmento anterior, 8 pacientes apresentaram múltiplos fragmentos, dos quais 3 pacientes com corpo estranho de vidro e os demais metálicos, em diferentes locais do segmento anterior sendo, caso 1: íris e cristalino (vidro), caso 2: seio camerular e corpo ciliar (vidro), caso 3: córnea, esclera e íris (vidro), caso 4: córnea e íris (metal) (Figura 1), caso 5: conjuntiva e câmara anterior (metal), casos 6 a 8: dois a cinco fragmentos na conjuntiva (metal)

Tais fragmentos de corpos estranhos no segmento anterior foram contabilizados junto aos demais encontrados, conforme sua localização: 11 na córnea (8 únicos e 3 dos múltiplos) (Figura 1); 10 na conjuntiva (6 únicos e 4 dos múltiplos); 10 na íris (7 únicos e 3 dos múltiplos) (Figuras 1 e 2); 9 no cristalino (7 únicos e 2 dos múltiplos); 5 na esclera (4 únicos e 1 dos múltiplos); 5 no seio camerular (4 únicos com 1 dos múltiplos); 3 no corpo ciliar (2 únicos e 1 dos múltiplos). 0 gráfico 1 representa a distribuição dos corpos estranhos de segmen-
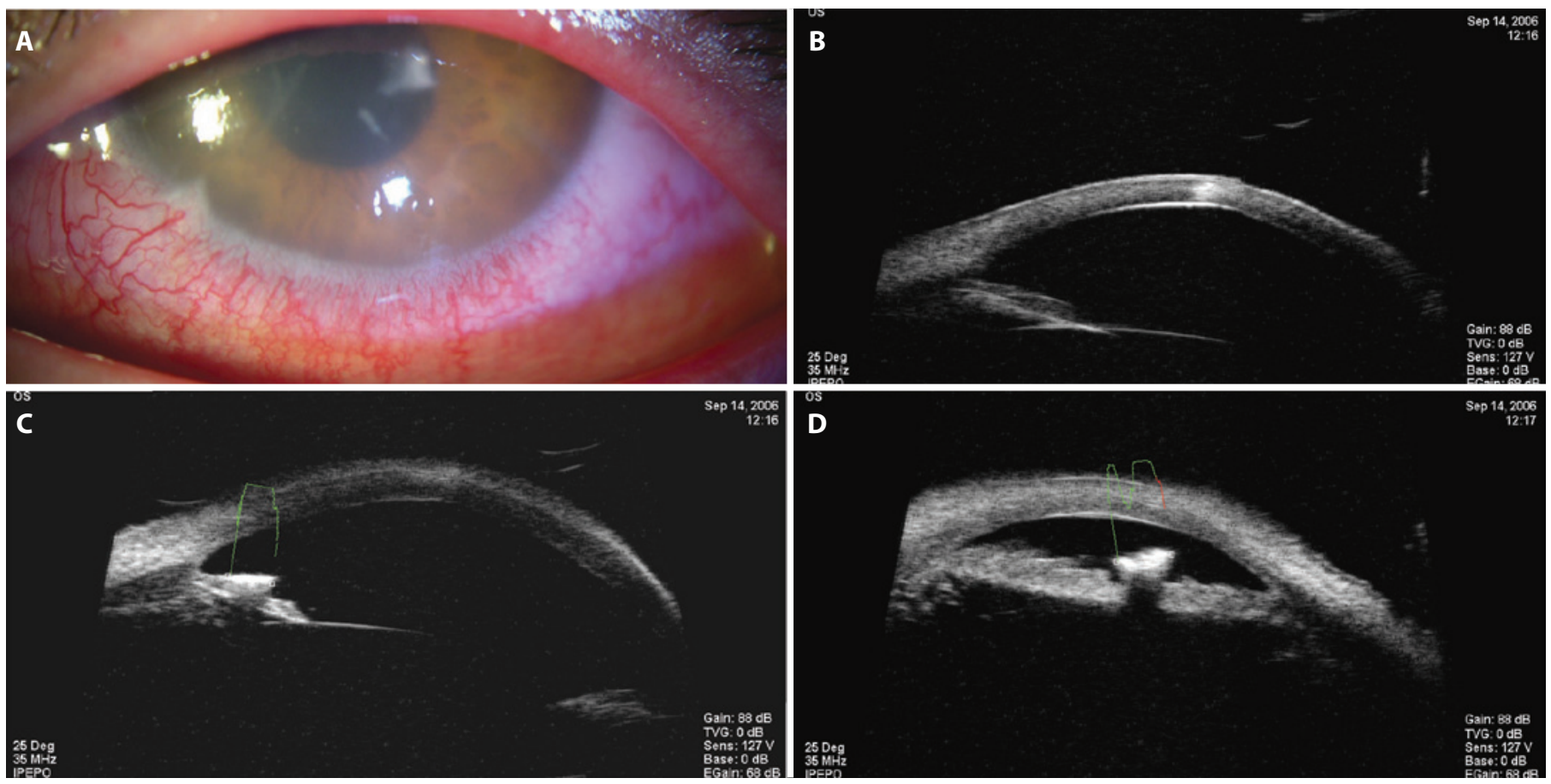

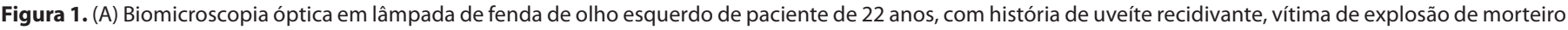

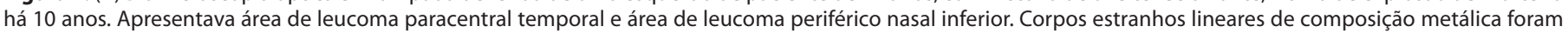

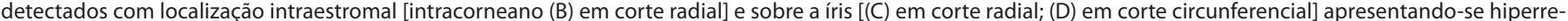

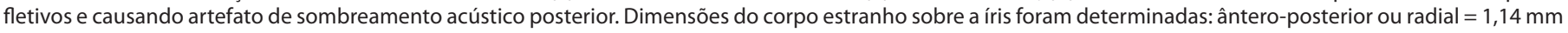
e látero-lateral ou circunferencial ou transversal =1,47 mm. 

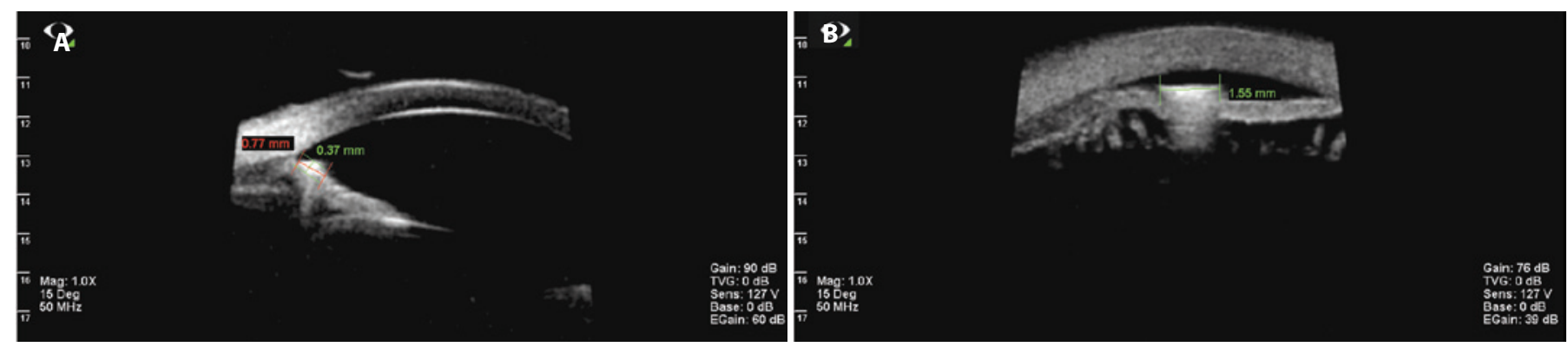

Figura 2. Ultrassonografia biomicroscópica (transdutor de $50 \mathrm{MHz}$, técnica de imersão) em paciente masculino de 33 anos, com história prévia de traumatismo ocular pérfurocontuso, demonstra presença de um corpo estranho alojado no segmento anterior do olho. O corpo estranho caracteriza-se por uma interface linear hiperrefletiva posicionada na íris e que gera múltiplas reflexões posteriores, artefato denominado duplicação ou reverberação. Suas dimensões foram determinadas em cortes radiais (A) e circunferenciais ou transversais $(B)$ espessura $=0,37 \mathrm{~mm}$; ântero-posterior ou radial $=0,77 \mathrm{~mm}$ e látero-lateral ou transversal ou circunferencial: 1,55 mm

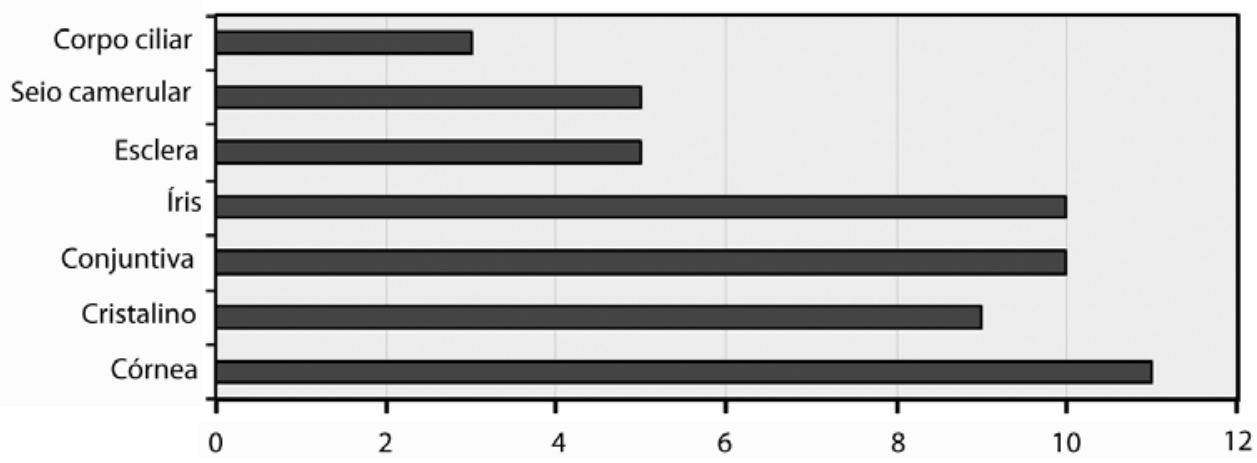

Gráfico 1. Localização dos corpos estranhos no segmento anterior ocular nos pacientes examinados com ultrassonografia biomicroscópica.

to anterior em relação à sua localização. A figura 2 mostra um caso de corpo estranho alojado no segmento anterior, sobre a íris.

Dentre as lesões associadas, pôde-se identificar: perfuração corneana em 12 casos, laceração corneana em 2 casos, sinéquia anterior em 6 casos, aniridia traumática em 1 caso, iridotomia traumática em 3 casos, reação de câmara anterior em 10 casos e rotura de cápsula anterior do cristalino em 4 casos.

A porta de entrada dos corpos estranhos foi identificada em 22 $(52,38 \%)$ casos e dentre estes, $18(81,81 \%)$ adentraram o olho pela córnea e 4 (18,19\%) pela conjuntiva. Foram contabilizados 27 corpos estranhos intraoculares: 10 na íris, 9 no cristalino, 5 no seio camerular e 3 no corpo ciliar. A UBM não foi capaz de identificar o trajeto de 5 corpos estranhos, pois caso o corpo estranho tenha penetrado pela esclera com pouca alteração da morfologia, há dificuldade na identificação da porta de entrada. Por vezes, devido à aposição de margens da ferida de entrada, casos de ferimentos de pequeno porte com mínima alteração anatômica do segmento anterior e após maior tempo de ocorrência do traumatismo, torna-se difícil identificar com exatidão o sítio de entrada pela técnica de UBM, um exame dinâmico que exige colaboração do paciente ao movimentar o olho acometido durante o exame de imersão para permitir a varredura cuidadosa de todos os meridianos. O tamanho dos 20 fragmentos que puderam ser medidos variou de 0,09 $\mathrm{mm}$ a 2,45 $\mathrm{mm}$, independente do local da medida (transversal ou radial), com média de 0,84 mm. Quanto à natureza, 21 (50\%) eram metálicos, 21 (50\%) eram não metálicos (20 identificados como vidro e 1 como vegetal).

\section{DISCUSSÃO}

Comparando este estudo com Magdaleno et al. verificou-se que, independente do momento histórico que o estudo foi realizado, a maioria dos pacientes de ambos estudos eram homens, com média de idade de 37,49 anos (idade laborativa), que os corpos estranhos do segmento anterior do olho eram, na maioria, de natureza metálica, e tiveram como porta de entrada a córnea(4).

Pode-se dizer que, para preservar os olhos dos pacientes que sofreram traumatismos com corpos estranhos, deve-se anteceder ao ato terapêutico com exames de imagem que sejam capazes de melhorar o prognóstico do olho afetado permitindo localização e dimensionamento do corpo estranho para garantir adequado planejamento cirúrgico, de forma que o prejuízo à visão seja reduzido.

O emprego da UBM para exploração do segmento anterior não é possível em olhos com perfuração ocular não tratada, exige colaboração do paciente, devendo-se ressaltar a dor no quadro de traumatismo recente. No entanto, há casos em que o exame clínico não permite determinar a localização do corpo estranho, por exemplo, em caso de opacidade de meios (coleção hemorrágica ou inflamatória do segmento anterior, edema de córnea) ou no alojamento do corpo estranho posteriormente a uma estrutura pigmentada ou opaca (retroiriano, por exemplo). Infelizmente neste estudo não foi possível acessar os exames clínicos dos pacientes, devido ao fato de ter sido um estudo retrospectivo e da indisponibilidade dos prontuários, visto que os casos vieram encaminhados do pronto socorro, em atendimento de urgência.

Este exame permitiu a identificação, caracterização, determinação do tamanho e da localização dos corpos estranhos metálicos ou não metálicos. Também foi capaz de determinar a presença de lesões associadas como a rotura do cristalino e da íris, além de fornecer a informação que permitiu o planejamento terapêutico, seja observação seriada, intervenção cirúrgica ou o tratamento isolado das lesões associadas do segmento anterior. 


\section{CONCLUSÕES}

A ultrassonografia biomicroscópica (UBM) auxiliou a localizar corpos estranhos no segmento anterior, não detectáveis por outros métodos inclusive devido à opacidade de meios. Pela UBM pôde-se diagnosticar ou supor a natureza dos corpos estranhos, esclarecer o seu trajeto e determinar a sua localização, facilitando a remoção cirúrgica quando necessária, sem causar mais lesões ao globo ocular.

\section{REFERÊNCIAS}

1. Cillino S, Casuccio A, Di Pace F, Pillitteri F, Cillino G. A five-year retrospective study of the epidemiological characteristics and visual outcomes of patients hospitalized for ocular trauma in a Mediterranean area. BMC Ophthalmol. 2008:8:6.

2. Hersh P, Zagelbaum B, Kenyon KB, Shingleton BJ. Surgical management of anterior segment trauma. In: Duane TD, Jaeger EA, editors. Duane`s Clinical ophthalmology. Philadelphia: Harper and Row; 2000. Vol 6, Chapter 39, p.11-4

3. Laroche D, Ishikawa H, Greenfield D, Liebmann JM, Ritch R. Ultrasound biomicroscopic localization and evaluation of intraocular foreign bodies. Acta Ophthalmol Scand. 1998;76(4):491-5

4. Magdaleno RL, Allemann N, Freitas D. Diagnóstico de corpos estranhos intraoculares pela biomicroscopia ultra-sônica [abstract]. Arq Bras Oftalmol. 1997;60(4):387.

5. Ishikawa H, Schuman JS. Anterior segment imaging: ultrasound biomicroscopy. Ophthalmol Clin North Am. 2004;17(1):7-20.

6. Deramo VA, Shah GK, Baumal CR, Fineman MS, Correa ZM, Benson WE, et al. The role of ultrasound biomicroscopy in ocular trauma. Trans Am Ophthalmol Soc. 1998;96: 355-65; discussion 365-7.

7. Atta HR. High frequency ultrasound. Br J Ophthalmol. 1995;79(11):967-8. Comment on Br J Ophthalmol. 1995;79(11):972-6.

8. Dorairaj S, Liebmann JM, Ritch R. Quantitative evaluation of anterior segment parameters in the era of imaging. Trans Am Ophthalmol Soc. 2007;105:99-108; discussion 108-10.

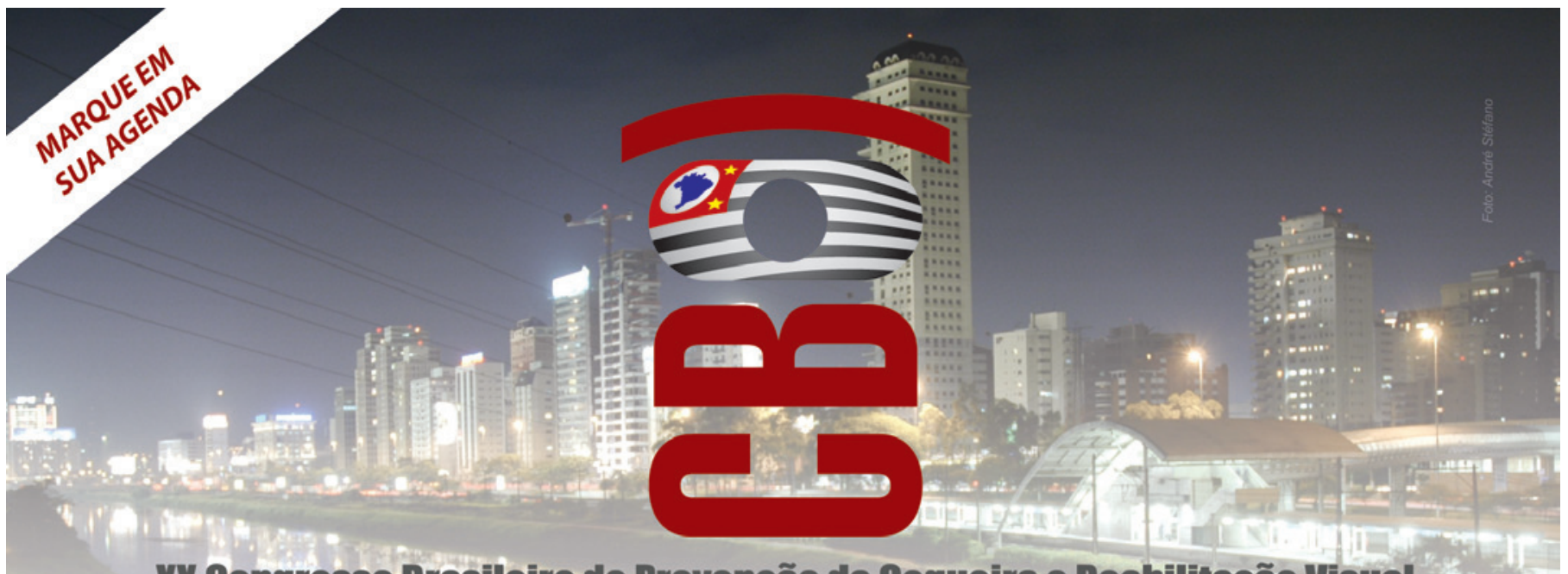

X Rongresso Brasileiro de Prevenção da Regueira e Reahilitação Visual

Gumbre de Las Americas para la Provención de la Beguera

12 a 15 de setembro de 2012

Anhembi - São Paulo - Brasil

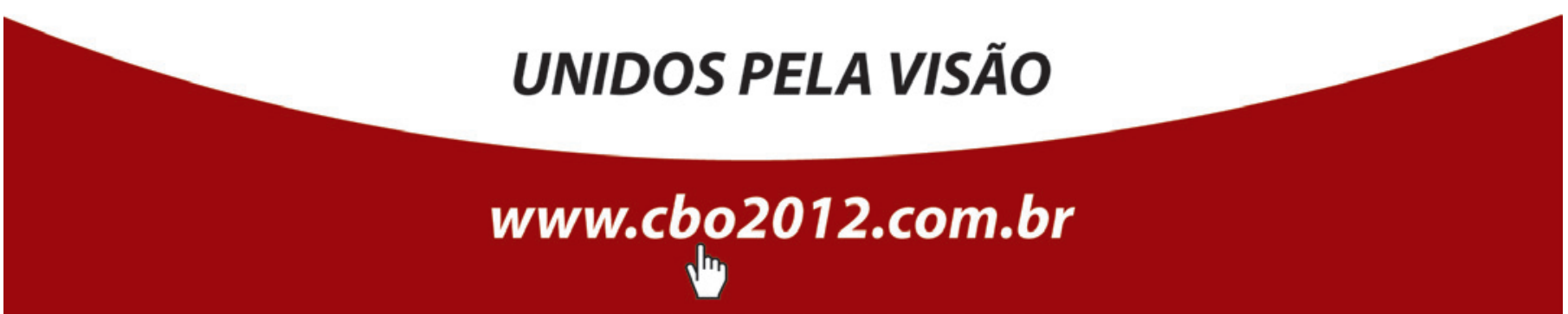

